\title{
FAM-FACE-SG: a score for risk stratification of frequent hospital admitters
}

\author{
Lian Leng Low ${ }^{1,2^{*}+}$, Nan Liu ${ }^{3,4^{*}}$, Kheng Hock Lee ${ }^{1,2}$, Marcus Eng Hock Ong ${ }^{5,6}$, Sijia Wang ${ }^{7}$, Xuan Jing ${ }^{3}$
} and Julian Thumboo 3,8

\begin{abstract}
Background: An accurate risk stratification tool is critical in identifying patients who are at high risk of frequent hospital readmissions. While 30-day hospital readmissions have been widely studied, there is increasing interest in identifying potential high-cost users or frequent hospital admitters. In this study, we aimed to derive and validate a risk stratification tool to predict frequent hospital admitters.

Methods: We conducted a retrospective cohort study using the readily available clinical and administrative data from the electronic health records of a tertiary hospital in Singapore. The primary outcome was chosen as three or more inpatient readmissions within 12 months of index discharge. We used univariable and multivariable logistic regression models to build a frequent hospital admission risk score (FAM-FACE-SG) by incorporating demographics, indicators of socioeconomic status, prior healthcare utilization, markers of acute illness burden and markers of chronic illness burden. We further validated the risk score on a separate dataset and compared its performance with the LACE index using the receiver operating characteristic analysis.
\end{abstract}

Results: Our study included 25,244 patients, with 70\% randomly selected patients for risk score derivation and the remaining $30 \%$ for validation. Overall, 4,322 patients (17.1\%) met the outcome. The final FAM-FACE-SG score consisted of nine components: Furosemide (Intravenous $40 \mathrm{mg}$ and above during index admission); Admissions in past one year; Medifund (Required financial assistance); Frequent emergency department (ED) use ( $\geq 3$ ED visits in 6 month before index admission); Anti-depressants in past one year; Charlson comorbidity index; End Stage Renal Failure on Dialysis; Subsidized ward stay; and Geriatric patient or not. In the experiments, the FAM-FACE-SG score had good discriminative ability with an area under the curve (AUC) of 0.839 (95\% confidence interval [Cl]: 0. 825-0.853) for risk prediction of frequent hospital admission. In comparison, the LACE index only achieved an AUC of $0.761(0.745-0.777)$.

Conclusions: The FAM-FACE-SG score shows strong potential for implementation to provide near real-time prediction of frequent admissions. It may serve as the first step to identify high risk patients to receive resource intensive interventions.

Keywords: Risk score, Stratification, Frequent hospital admitters, Electronic health record, LACE index

\footnotetext{
*Correspondence: low.lian.leng@singhealth.com.sg;

liu.nan@singhealth.com.sg

${ }^{\dagger}$ Equal contributors

'Department of Family Medicine \& Continuing Care, Singapore General

Hospital, Singapore, Singapore

${ }^{3}$ Health Services Research Centre, Singapore Health Services, Singapore,

Singapore

Full list of author information is available at the end of the article
}

(c) The Author(s). 2017 Open Access This article is distributed under the terms of the Creative Commons Attribution 4.0 International License (http://creativecommons.org/licenses/by/4.0/), which permits unrestricted use, distribution, and reproduction in any medium, provided you give appropriate credit to the original author(s) and the source, provide a link to the Creative Commons license, and indicate if changes were made. The Creative Commons Public Domain Dedication waiver (http://creativecommons.org/publicdomain/zero/1.0/) applies to the data made available in this article, unless otherwise stated. 


\section{Background}

Health systems worldwide continue to struggle with the rapidly ageing population and increasing chronic disease burden. One key focus to reduce over reliance on hospital care is to improve the care transitions of patients at high risk of readmissions or frequent hospital admissions. An accurate, validated readmission risk stratification tool is the critical first step to identify these high risk patients to receive resource intensive interventions. To date, almost all readmission risk stratification tools have focused on predicting 30-day readmissions [1, 2]. This is in line with section 3025 of the United States Affordable Care Act that mandated the Centers for Medicare and Medicaid services to penalize hospitals with excessive readmission rates for heart failure, acute myocardial infarction and pneumonia [3]. While readmissions are arguably most preventable in the immediate post-discharge period, there is a strong case to be made for developing sustainable integrated care programs with emphasis on longer term outcomes. There is increasing interest in identifying potential high-cost users or frequent hospital admitters [4-8] who account for a disproportionate amount of healthcare spending $[6,9]$. Moreover, there is significant overlap between frequent hospital admitters and 30-day re-admitters. Black et al. found that although patients with three or more readmissions accounted for only $10.2 \%$ of patients, these patients accounted for $71.6 \%$ of 30 -day readmissions [10]. Lack of coordinated programs to improve the care outcomes for these high risk patients risk a vicious spiral of increasing healthcare utilization and expenditure and poorer quality of life. In heart failure patients, it is well known that each episode of hospitalization is associated with an incrementally poorer prognosis and greater mortality [11]. Efforts to reduce avoidable readmissions need to look beyond the current focus on a single hospital discharge and transition period to a patient-centric focus that manages high risk patients as a patient segment.

Singapore is a developed city state in South East Asia with a population of 5.6 million. In 2014, Bloomberg ranked the Singapore healthcare system as the most efficient in the world, despite spending a relatively small proportion of gross domestic product on healthcare and having one of the lowest healthcare costs per capita [12]. Like most developed health systems struggling with a rapidly ageing population, increasing chronic disease burden and a shrinking workforce, the Singapore healthcare system has been transforming from a hospital centric model to a population centric model to avoid an unsustainable healthcare system. To achieve this, the healthcare system has been re-organized into regional health systems (RHS) that aimed to deliver value-based, patient centered care [8]. In each RHS, there is a primary tertiary hospital as well as a secondary hospital that provides intermediate and rehabilitation care; in its region, there are primary care and long-term care services to link vertical and horizontal integration of care.

Since 2015, each RHS receives capitated funding to improve the care transitions and reduce readmission risk of patients at high risk of frequent admissions in its catchment population. Frequent admitters or "familiar faces" in Singapore are defined as patients with three or more inpatient admissions in a year. These are high-cost patients, with an average cost per patient approaching SGD 30,000 a year [7]. In the SingHealth RHS, 80 senior nurses have been trained in transitional care as patient navigators [13] (PNs) to perform case management. Transitional care services have been re-organized into integrated practice units $[14,15]$ to coordinate the care of patients through the complex healthcare system for the one year after hospital discharge. These interventions include a comprehensive needs assessment, followed by case management, post-discharge monitoring [15] and home care [14]. These interventions are resource intensive and must be targeted at patients at highest risk of frequent admissions to be cost-effective and sustainable. An accurate and validated frequent hospital admission predictive risk score is critical for risk stratification. The risk score should ideally be automated for easy adoption by healthcare users and provide real-time/near real-time risk scores early in the admission in order to impact interventions before hospital discharge.

SingHealth RHS has a well-established electronic health record (EHR) system that was developed and implemented more than 10 years ago. The EHR integrates information from multiple sources including administrative data (for example, patient demographics), clinical data and ancillary, called the Electronic Health Intelligence System [16]. The Singapore system is comparable with the definition of EHR used in the US or Europe. On a daily basis, data from these multiple sources are extracted, transformed and loaded onto the enterprise data warehouse. Further use can be in the form of reports, dashboards and mobile applications to support business and operational needs. Leveraging on this well-established EHR system, it is possible to derive a near real-time (refreshed on a daily basis) automated predictive risk score to identify high risk patients for intervention. Currently, based on Kansagara's review [1] and more recent Zhou's review [17], around ten published predictive models utilized real-time administrative data.

Our study aims to address the lack of an accurate, validated frequent admitter risk score. Our primary objective is to derive a real-time frequent hospital admission risk stratification model to predict frequent hospital admitters or "familiar faces in Singapore". We will compare our model with the LACE index [18], an established risk stratification tool. The LACE index includes four components, namely length of stay ("L"), acuity of the admission ("A"), comorbidity of the patient ("C") and emergency 
department use ("E"). It can be easily calculated and has been widely used for predicting the risk of readmissions within 30 days after discharge of hospital. However, the LACE index demonstrated inconsistent performance [17]. Our study hypothesizes that by including potentially discriminatory predictors such as medications used during the hospitalization episode and indicators of socioeconomic status, the new risk stratification model will perform better than the LACE index at predicting frequent hospital admissions within 12 months of discharge.

\section{Methods}

\section{Study design and participants}

We conducted a retrospective observational cohort study using the EHR data of the Singapore General Hospital (SGH). This study was approved by SingHealth Centralized Institutional Review Board (CIRB 2015/2696) with waiver of informed consent. We included all admitted adult patients ( $\geq 21$ years of age, i.e., the legal age for majority in Singapore) from January 1, 2013 to May 31, 2014. For patients who were admitted multiple times, we only included the index admission. We excluded patients who were non-residents, died during the index admission, and whose admission specialty was obstetrics, emergency medicine, dentistry or ophthalmology. Emergency medicine admissions were excluded as these were observation ward admissions rather than true hospital admissions. Patients admitted to the emergency medicine observation ward are typically monitored up to $24 \mathrm{~h}$ and subsequently discharged home or converted to hospital admissions according to their clinical circumstances. Patients converted to hospital admissions would be captured in our dataset. We also excluded patients admitted to obstetrics as these admissions are pregnancy related; and admissions to dentistry and ophthalmology which are usually elective in nature.

\section{Outcome and variables}

The primary outcome in this study was chosen as three or more inpatient readmissions within 12 months of discharge. Candidate variables were selected by a group of clinical experts based on our preliminary study on frequent admissions [2] and existing literature [1, 16, 19, 20]. Our independent variables included patient demographics (age, gender and ethnicity), comorbidities, healthcare utilization in preceding year (number of admissions, number of specialist outpatient clinic visits, and number of emergency department visits), and variables reflecting socioeconomic status. Specifically, comorbidities (the Charlson and Elixhauser Comorbidity Index measures [21-23]) were identified using International Classification of Diseases Ninth and Tenth Revision (ICD-9 and ICD-10) codes for major diagnoses in the past seven years (as shown in Table 1). Our approach is among the most comprehensive in the literature [24] and would reduce potential lapses in diagnostic coding.

To assess the socioeconomic status, we used two indicators: whether the patient stayed in subsidized ward class during index admission and whether the patient was assisted by Medifund. In Singapore, hospitals use means testing to evaluate the patient's eligibility for subsidized wards. It is based on patients' income or the annual value of their residence. The government aims to share the limited subsidies to all Singaporeans in a fair manner by providing more subsidies to people with lower socioeconomic status. Additionally, those patients who are still unable to afford their healthcare bills after receiving government subsidies and other means of payments including insurance may apply for Medifund assistance. Medifund is a safety net uniquely provided by Singapore government to help patients with special financial difficulties [25]. The actual amount of assistance also depends on the patient's socioeconomic status.

We selected seven variables of acute illness burden potentially predictive of frequent admissions. As listed in Table 1, these variables included whether the index admission was urgent, whether second line antibiotics were required, whether treatment with intravenous furosemide $40 \mathrm{mg}$ or more was required, whether inpatient dialysis was required, length of stay of the index admission, and the Charlson Comorbidity Index (CCI) of index admission. One of the novelties in this study was to use the $\mathrm{CCI}$ in weighting the index discharge diagnoses, which was already a key marker for mortality within one year [22]. Intravenous furosemide was chosen as one of candidate variables because it has been used in acute treatment of heart failure and therefore a proxy marker of heart disease severity. Moreover, we found it a significant predictor of frequent hospital admission risk [2] and 30-day readmission risk [26].

Antidepressants play an important role in the treatment of depression and may be associated with hospital readmissions [27, 28]. We hypothesized that the use of antidepressants would affect the risk of frequent admissions. Patients with depression are more likely treated as outpatients and would not have discharge ICD codes for depression. Therefore, we included the variable of whether the patient had treatment with antidepressants in the past one year in our list of predictors.

\section{Model derivation and validation}

We randomly selected $70 \%$ of the data for model derivation and the remaining $30 \%$ of the data for model validation. Due to low prevalence rate of frequent hospital admitters, we split the data by strata, that is, we randomly selected equal percentage of data from both frequent admitters group and non-frequent admitters group into both derivation and validation sets. We conducted univariable and 


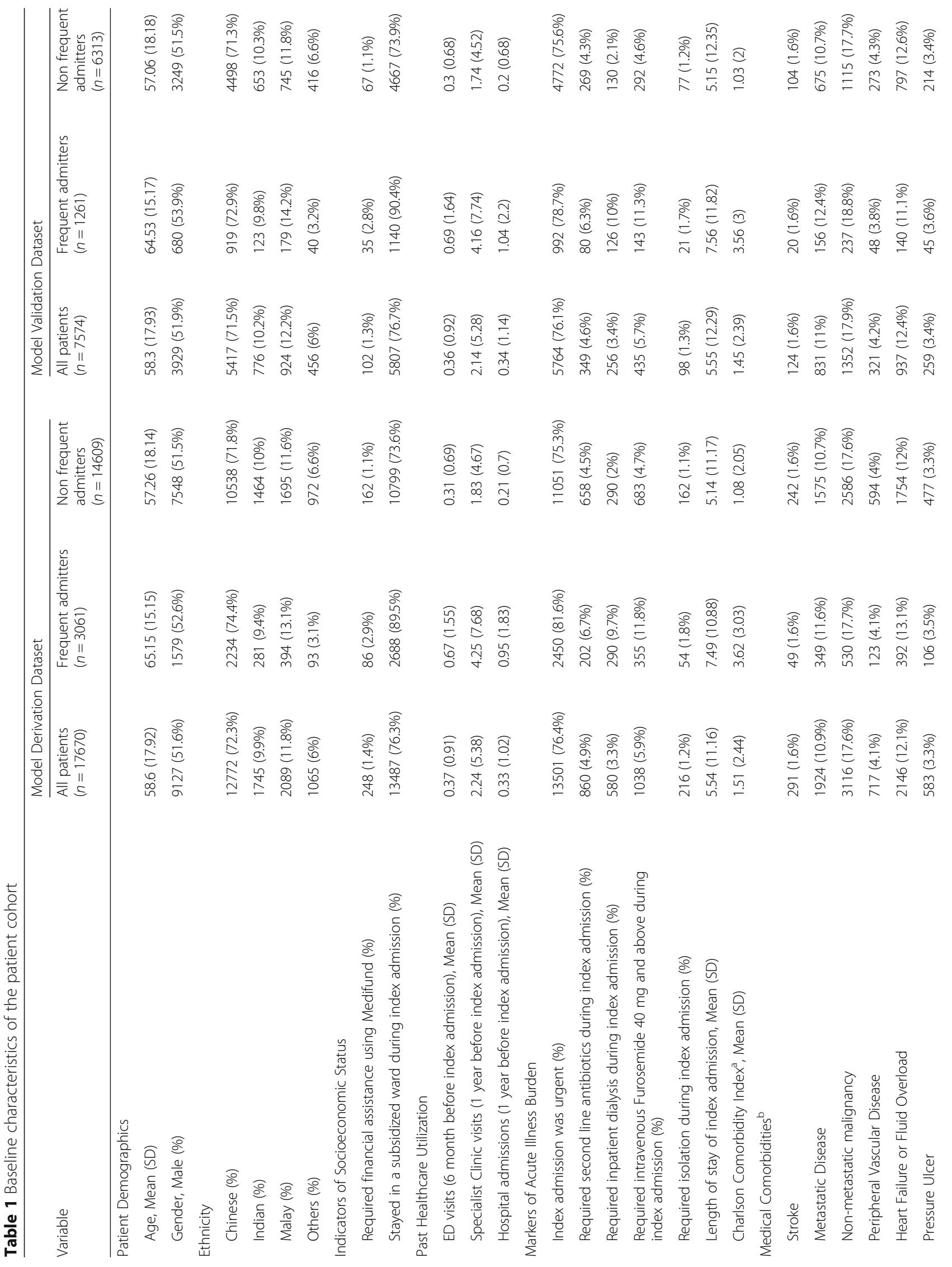




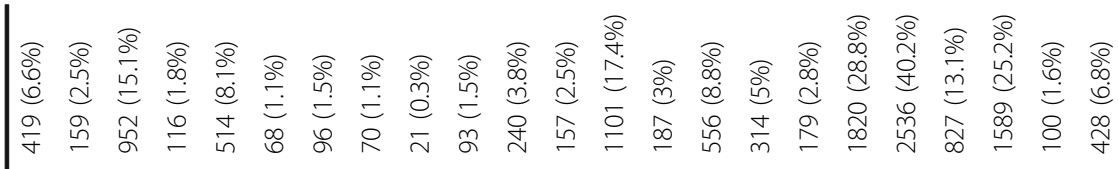

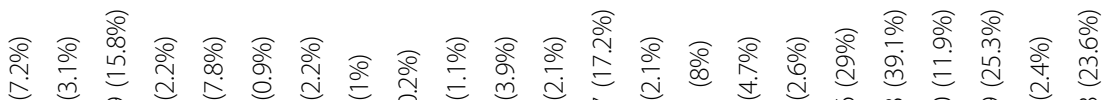

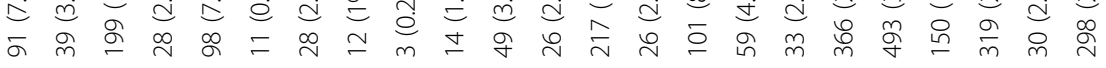

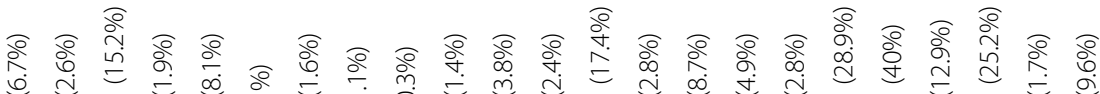

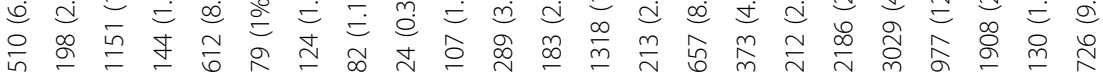

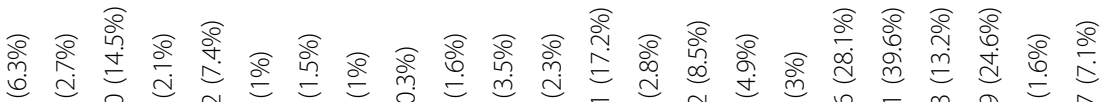
反.

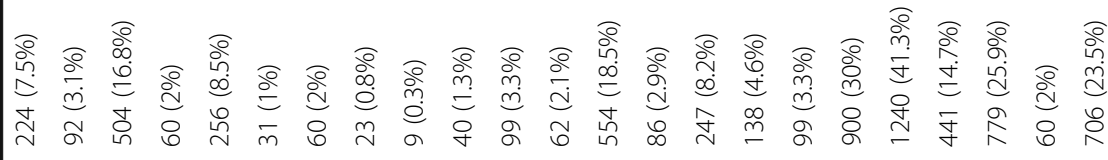

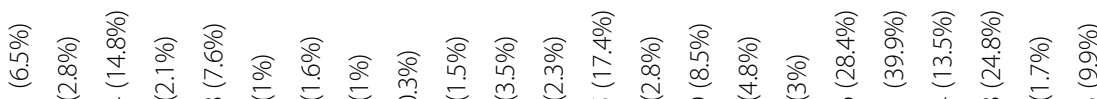

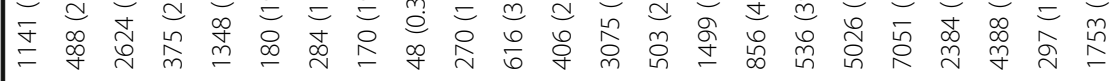

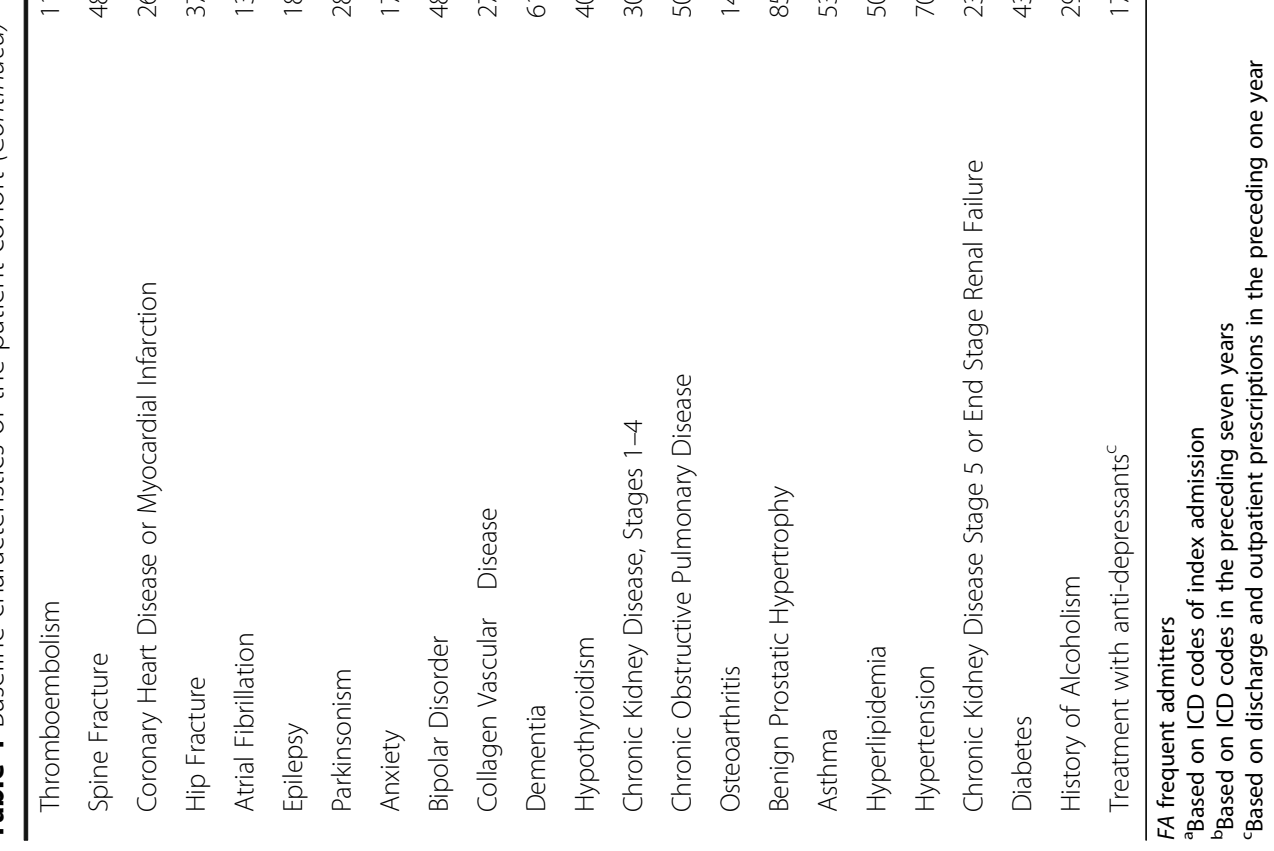


multivariable logistic regression to measure the independent association of predictive variables with hospital frequent admission. We chose the most significant $(p<$ $0.02)$ predictive variables and used clinical knowledge to determine a set of candidate variables for final model derivation. We adopted a popular risk score derivation method $[18,29]$ to convert the final logistic model into a risk index. The principle to derive the risk point for each variable was to divide its regression $\beta$ coefficient by the smallest $\beta$ coefficients of all variables, and to round the value to the nearest whole number. Then, the final score was calculated by summing up the risk points of all variables.

We assessed model discrimination using the validation set, that is, randomly selected $30 \%$ of the data. We conducted the receiver operating characteristic (ROC) analysis to evaluate the performance of the predictive model. From the ROC analysis, we also computed measures of diagnostic accuracy such as sensitivity, specificity, positive predictive value (PPV), and negative predictive value (NPV). We performed all analyses using $\mathrm{R}$ version 3.2.3 (R Foundation, Vienna, Austria).

\section{Results}

From January 1, 2013 to May 31, 2014, a total of 206,699 patient admissions were recorded in SGH. Among all records, we excluded 111,312 non-resident patient cases, 12,252 patient cases whose admission specialty was obstetrics, emergency medicine, dentistry or ophthalmology, and 4,157 patient cases who died during the hospitalization. With the remaining 74,102 adult patient cases, we further excluded 31,813 patient cases that have not completed one year follow-up at the time of analysis and 17,045 cases of non-index admissions. Overall, 25,244 patients were included in this study. Of these, 4,322 patients (17.1\%) had three or more admissions in the one year period following index discharge (Fig. 1). These patients were classified as frequent admitters according to our definition. The final model contained nine significant predictors (Furosemide intravenous $40 \mathrm{mg}$ and above; Admissions in the past one year; Medifund; Frequent Emergency Department use $\geq 3$ in the past six months; Anti-depressants in past one year; Charlson Comorbidity Index; End stage renal failure on dialysis; Subsidized ward stay; and Geriatric patient). Accordingly, we termed the novel index as the FAMFACE-SG score.

Table 1 tabulates the baseline characteristics of the study population. The mean age of frequent admitters was 65 (standard deviation $[\mathrm{SD}]=15$ ) years and the mean age of non-frequent admitters was $57(\mathrm{SD}=18)$ years. Male patients accounted for $51.7 \%$ of the total study population. Of all index admissions, 76.3\% $(n=19,265)$ were urgent. The mean length of stay of index admission was 5.54 days. On average, frequent admitters had a mean CCI of 3.60 and non-frequent admitters had a mean CCI of 1.06. Compared to other patients, frequently admitted patients were more likely to be older, required a significantly longer stay for their index admission, had a higher CCI score, and had more ED admissions in the preceding six months.

The results of univariable and multivariable logistic regression analysis on frequent hospital admissions are presented in Table 2. Nine variables were found to be significantly associated with frequent hospital admissions. As shown in Table 3, these variables included age, required financial assistance using Medifund, stayed in a subsidized ward class during index admission, number of ED visits within the past six months before index admission, number of hospital admissions within the past one year before index admission, required dialysis during index admission, treatment with intravenous Furosemide $40 \mathrm{mg}$ and above during index admission, Charlson Comorbidity Index, and treatment with antidepressants in the past one year. The top two strongest independent predictors were admission to a subsidized ward class during index admission (adjusted OR: 4.05, 95\% confidence interval [CI]: 2.546.85) and treatment with antidepressants in the past one year (adjusted odds ratio [OR]: 2.33, 95\% CI: 2.062.64). Additionally, patients who required financial assistance using Medifund were 1.49 times more likely (adjusted OR: $1.49,95 \%$ CI: $1.08-2.04)$ to become a FA. Every increase of one point on the CCI score was associated with $31 \%$ increased risk of becoming a FA (adjusted OR: 1.31, 95\% CI: 1.28-1.33).

Figure 2 shows the ROC curve of the final logistic regression model. Using the proposed FAM-FACE-SG score, the final predictive model had better discriminative ability than the traditional LACE index (area under the curve [AUC]: 0.839, 95\% CI: 0.825-0.853). The optimal cut-off for the regression model was 13, which achieved a sensitivity of $81.6 \%$ (95\% CI: $79.5-83.7 \%$ ) and a specificity of $73.3 \%(72.2-74.4 \%)$. In addition, Table 4 shows the sensitivity, specificity, PPV and NPV according to these cut-offs.

\section{Discussion}

In this study, we proposed a risk stratification model (FAM-FACE-SG) by incorporating demographics, indicators of socioeconomic status, prior healthcare utilization, markers of acute illness burden and markers of chronic illness burden for the prediction of frequent hospital admission risk in Singapore. In model validation, FAMFACE-SG significantly outperformed the LACE index in terms of AUC $(0.839$ vs $0.761, p<0.001)$. In addition, FAM-FACE-SG performs better than the LACE index in achieving higher sensitivity, specificity, positive predictive value, and negative predictive value. The LACE index had functioned as a benchmark for predicting 30-day readmission risk due to its popularity and simplicity in 


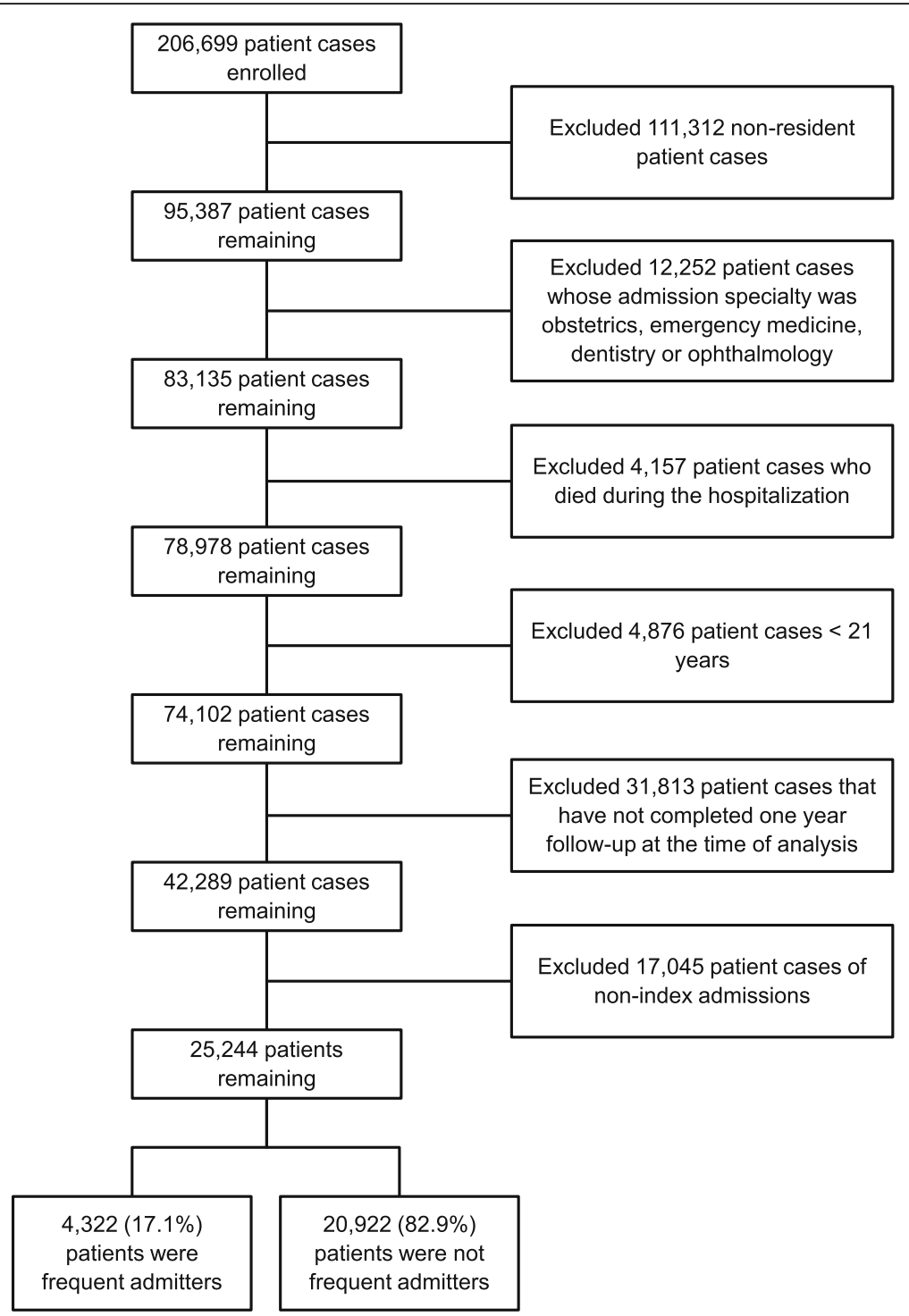

Fig. 1 Patient selection in the study

computation. The FAM-FACE-SG score overcomes the disadvantages of the LACE index with its near realtime availability early in the admission.

This study is novel by being the first study to derive and internally validate a risk score for frequent hospital admission risk. We considered well established predictors for readmission risk such as demographics, indicators of socioeconomic status, and prior healthcare utilization $[1,8,30]$ in deriving the score. Developed in close collaboration between data scientists and clinicians who are involved in transitional care of patients, the score also incorporated clinical variables such as the use of anti-depressants as a proxy measure of clinically significant depression. We believe that beyond improving predictive performance of the tool, such variables may help us identify patients who are more amenable to clinical interventions.
In addition, we incorporated markers of acute illness burden such as intravenous furosemide and dialysis into FAM-FACE-SG risk score for near real-time prediction. Our work highlights the excellent opportunity to leverage on the technological advancements in EHR and business analytics tools to generate real-time insights that support clinical care. In constructing the FAM-FACE-SG score, Charlson comorbidity index and admissions in the past one year contribute the greatest weight to the final score. While it would be ideal to achieve parsimony of the risk score by excluding Medifund and Subsidized ward stay, placed in the national context of deriving a risk stratification score to identify high risk patients as a critical first step in transitional care programs, we believe it is important to report the impact of indicators of socioeconomic status on frequent hospital admission risk. 
Table 2 Univariable and multivariable logistic regression

\begin{tabular}{|c|c|c|c|c|}
\hline Variable & Unadjusted OR (95\% Cl) & $p$-value & Adjusted OR (95\% Cl) & $p$-value ${ }^{*}$ \\
\hline \multicolumn{5}{|l|}{ Patient Demographics } \\
\hline Age & $1.03(1.02,1.03)$ & $<0.001$ & $1.01(1.01,1.017)$ & $<0.001^{\dagger}$ \\
\hline Gender (Male) & $1.05(0.97,1.13)$ & 0.254 & $1.02(0.93,1.12)$ & 0.671 \\
\hline \multicolumn{5}{|l|}{ Ethnicity } \\
\hline Others & Baseline & & & \\
\hline Chinese & $1.14(1.04,1.25)$ & 0.004 & $0.90(0.70,1.16)$ & 0.407 \\
\hline Indian & $0.93(0.81,1.06)$ & 0.300 & $1.18(0.89,1.59)$ & 0.249 \\
\hline Malay & $1.16(1.03,1.30)$ & 0.015 & $1.04(0.79,1.39)$ & 0.764 \\
\hline \multicolumn{5}{|l|}{ Indicators of Socioeconomic Status } \\
\hline Required financial assistance using Medifund & $2.64(2.02,3.43)$ & $<0.001$ & $1.49(1.08,2.04)$ & $0.015^{\dagger}$ \\
\hline Stayed in a subsidized ward during index admission & $3.07(2.72,3.47)$ & $<0.001$ & $4.05(2.54,6.85)$ & $<0.001^{\dagger}$ \\
\hline \multicolumn{5}{|l|}{ Past Healthcare Utilization } \\
\hline ED visits (6 month before index admission) & $1.47(1.41,1.53)$ & $<0.001$ & $1.15(1.09,1.22)$ & $<0.001^{\dagger}$ \\
\hline Specialist Clinic visits (1 year before index admission) & $1.07(1.06,1.07)$ & $<0.001$ & $1.01(1.00,1.02)$ & 0.088 \\
\hline Hospital admissions (1 year before index admission) & $1.77(1.70,1.84)$ & $<0.001$ & $1.32(1.26,1.39)$ & $<0.001^{\dagger}$ \\
\hline \multicolumn{5}{|l|}{ Markers of Acute Illness Burden } \\
\hline Index admission was urgent & $1.45(1.32,1.61)$ & $<0.001$ & $0.92(0.73,1.16)$ & 0.465 \\
\hline Required second line antibiotics during index admission & $1.54(1.30,1.80)$ & $<0.001$ & $1.07(0.86,1.32)$ & 0.534 \\
\hline Required inpatient dialysis during index admission & $5.30(4.48,6.27)$ & $<0.001$ & $1.72(1.38,2.15)$ & $<0.001^{\dagger}$ \\
\hline Required intravenous Furosemide $40 \mathrm{mg}$ and above during index admission & $2.75(2.40,3.14)$ & $<0.001$ & $1.26(1.07,1.48)$ & $0.005^{\dagger}$ \\
\hline Required isolation during index admission & $1.64(1.19,2.22)$ & 0.002 & $1.19(0.81,1.72)$ & 0.375 \\
\hline Length of stay of index admission & $1.02(1.01,1.02)$ & $<0.001$ & $1.00(1.00,1.00)$ & 0.919 \\
\hline Charlson Comorbidity Index ${ }^{a}$ & $1.41(1.38,1.42)$ & $<0.001$ & $1.31(1.28,1.33)$ & $<0.001^{\dagger}$ \\
\hline \multicolumn{5}{|l|}{ Medical Comorbidities ${ }^{b}$} \\
\hline Stroke & $0.99(0.72,1.34)$ & 0.945 & $0.93(0.64,1.34)$ & 0.712 \\
\hline Metastatic Disease & $1.09(0.97,1.24)$ & 0.154 & $1.07(0.90,1.28)$ & 0.43 \\
\hline Non-metastatic malignancy & $1.00(0.90,1.11)$ & 0.973 & $0.96(0.83,1.11)$ & 0.569 \\
\hline Peripheral Vascular Disease & $1.01(0.83,1.23)$ & 0.904 & $1.10(0.86,1.40)$ & 0.456 \\
\hline Heart Failure or Fluid Overload & $1.11(0.98,1.24)$ & 0.093 & $1.00(0.84,1.19)$ & 0.978 \\
\hline Pressure Ulcer & $1.09(0.88,1.34)$ & 0.435 & $1.14(0.88,1.48)$ & 0.307 \\
\hline Thromboembolism & $1.21(1.04,1.41)$ & 0.014 & $1.21(1.00,1.46)$ & 0.045 \\
\hline Spine Fracture & $1.14(0.90,1.43)$ & 0.266 & $1.05(0.79,1.38)$ & 0.743 \\
\hline Coronary Heart Disease or Myocardial Infarction & $1.19(1.07,1.33)$ & 0.001 & $1.1(0.94,1.29)$ & 0.225 \\
\hline Hip Fracture & $0.93(0.70,1.22)$ & 0.607 & $0.96(0.69,1.32)$ & 0.795 \\
\hline Atrial Fibrillation & $1.16(1.00,1.33)$ & 0.042 & $1.1(0.92,1.32)$ & 0.302 \\
\hline Epilepsy & $1.02(0.68,1.48)$ & 0.933 & $0.82(0.51,1.28)$ & 0.394 \\
\hline Parkinsonism & $1.32(0.98,1.74)$ & 0.062 & $1.43(1.01,2.00)$ & 0.043 \\
\hline Anxiety & $0.76(0.48,1.16)$ & 0.229 & $0.57(0.33,0.92)$ & 0.028 \\
\hline Bipolar Disorder & $1.13(0.51,2.23)$ & 0.745 & $1.18(0.48,2.65)$ & 0.699 \\
\hline Collagen Vascular Disease & $0.85(0.60,1.17)$ & 0.338 & $0.76(0.51,1.12)$ & 0.175 \\
\hline Dementia & $0.93(0.75,1.16)$ & 0.537 & $0.91(0.70,1.18)$ & 0.498 \\
\hline Hypothyroidism & $0.88(0.66,1.15)$ & 0.352 & $0.93(0.67,1.28)$ & 0.681 \\
\hline Chronic Kidney Disease, Stages 1-4 & $1.09(0.98,1.21)$ & 0.095 & $0.97(0.79,1.18)$ & 0.731 \\
\hline Chronic Obstructive Pulmonary Disease & $1.01(0.79,1.27)$ & 0.947 & $1.07(0.80,1.41)$ & 0.648 \\
\hline
\end{tabular}


Table 2 Univariable and multivariable logistic regression (Continued)

\begin{tabular}{|c|c|c|c|c|}
\hline Osteoarthritis & $0.96(0.83,1.11)$ & 0.582 & $0.90(0.75,1.07)$ & 0.223 \\
\hline Benign Prostatic Hypertrophy & $0.94(0.77,1.12)$ & 0.489 & $0.95(0.76,1.18)$ & 0.619 \\
\hline Asthma & $1.11(0.89,1.38)$ & 0.354 & $1.22(0.93,1.59)$ & 0.152 \\
\hline Hyperlipidemia & $1.09(1.00,1.19)$ & 0.04 & $0.99(0.85,1.14)$ & 0.868 \\
\hline Hypertension & $1.07(0.99,1.16)$ & 0.085 & $0.96(0.83,1.11)$ & 0.587 \\
\hline Chronic Kidney Disease Stage 5 or End Stage Renal Failure & $1.13(1.01,1.26)$ & 0.035 & $1.03(0.83,1.28)$ & 0.81 \\
\hline Diabetes & $1.07(0.98,1.17)$ & 0.12 & $1.03(0.90,1.17)$ & 0.71 \\
\hline History of Alcoholism & $1.24(0.93,1.64)$ & 0.138 & $0.91(0.64,1.27)$ & 0.582 \\
\hline Treatment with anti-depressants ${ }^{c}$ & $4.00(3.60,4.44)$ & $<0.001$ & $2.33(2.06,2.64)$ & $<0.001^{\dagger}$ \\
\hline
\end{tabular}

$O R$ odds ratio, $E D$ emergency department, $I C D$ international classification of diseases

* Statistical significance was set as $p<0.02$

${ }^{\dagger}$ Variables selected for building final logistic regression model

a Based on ICD codes of index admission

${ }^{b}$ Based on ICD codes in the preceding seven years

${ }^{\mathrm{C}}$ Based on discharge and outpatient prescriptions in the preceding one year

Risk stratification enables the identification of individuals at risk of an unwanted outcome and effect targeted interventions for optimal results. Most transitional care interventions are aimed at preventing re-admissions and reducing inappropriate utilization of expensive hospital services. The optimization of transitional care requires the re-organization of care processes. Sometimes additional resources such as case managers are required. Improving outcome through transitional care must therefore be achieved at equal or lower cost of care. A clinically reliable tool that can guide the intervention towards patients who are at highest risk of suffering from suboptimal care transition is therefore key to making such programs cost effective. The quest for a good risk prediction tool for transitional care requires it to be low cost, technically accurate and clinically practical. Tools that use data that is not routinely captured during usual care of patients will add cost and increase the administrative burden of healthcare workers. Our tool was derived using administrative and clinical data that is routinely captured during care of hospitalized patients. This averted the need for additional cost and the work of data

Table 3 Predictors for deriving the FAM-FACE-SG score

\begin{tabular}{|c|c|c|c|c|c|c|}
\hline Predictor & Value & OR $(95 \% \mathrm{Cl})$ & $p$-value & $\beta$ Coefficients & Normalized $\beta$ Coefficients & Final Score \\
\hline $\begin{array}{l}\text { Furosemide (Intravenous } 40 \mathrm{mg} \text { and above } \\
\text { during index admission) }\end{array}$ & Yes & $1.26(1.09,1.47)$ & 0.002 & 0.2311 & 1.8912 & 2 \\
\hline \multirow[t]{4}{*}{ Admissions in past one year } & 0 & & & - & - & 0 \\
\hline & $1-2$ & $1.87(1.66,2.10)$ & $<0.001$ & 0.6259 & 5.1219 & 5 \\
\hline & $3-4$ & $3.5(2.79,4.40)$ & $<0.001$ & 1.2528 & 10.252 & 10 \\
\hline & $>4$ & $5.31(3.82,7.45)$ & $<0.001$ & 1.6696 & 13.6628 & 14 \\
\hline Medifund (Required financial assistance) & Yes & $1.7(1.25,2.30)$ & 0.001 & 0.5306 & 4.3421 & 4 \\
\hline $\begin{array}{l}\text { Frequent ED use ( } \geq 3 \text { ED visits in } 6 \text { month } \\
\text { before index admission) }\end{array}$ & Yes & $1.59(1.22,2.07)$ & 0.001 & 0.4637 & 3.7946 & 4 \\
\hline Anti-depressants in past one year & Yes & $2.42(2.14,2.73)$ & $<0.001$ & 0.8838 & 7.2324 & 7 \\
\hline \multirow[t]{5}{*}{ Charlson Comorbidity Index } & 0 & & & - & - & 0 \\
\hline & 1 & $2.5(2.11,2.95)$ & $<0.001$ & 0.9163 & 7.4984 & 7 \\
\hline & 2 & $5.11(4.48,5.83)$ & $<0.001$ & 1.6312 & 13.3486 & 13 \\
\hline & 3 & $7.86(6.55,9.42)$ & $<0.001$ & 2.0618 & 16.8723 & 17 \\
\hline & $\geq 4$ & $11.75(10.37,13.33)$ & $<0.001$ & 2.4639 & 20.1628 & 20 \\
\hline End Stage Renal Failure on Dialysis & Yes & $1.65(1.36,2.00)$ & $<0.001$ & 0.5008 & 4.0982 & 4 \\
\hline Subsidized ward stay & Yes & $1.5(1.31,1.71)$ & $<0.001$ & 0.4055 & 3.3183 & 3 \\
\hline \multirow[t]{3}{*}{ Geriatric patient (Age) } & $<65$ & & & - & - & 0 \\
\hline & $65-84$ & $1.13(1.03,1.25)$ & 0.013 & 0.1222 & 1 & 1 \\
\hline & $\geq 85$ & $1.43(1.21,1.68)$ & $<0.001$ & 0.3577 & 2.9272 & 3 \\
\hline
\end{tabular}




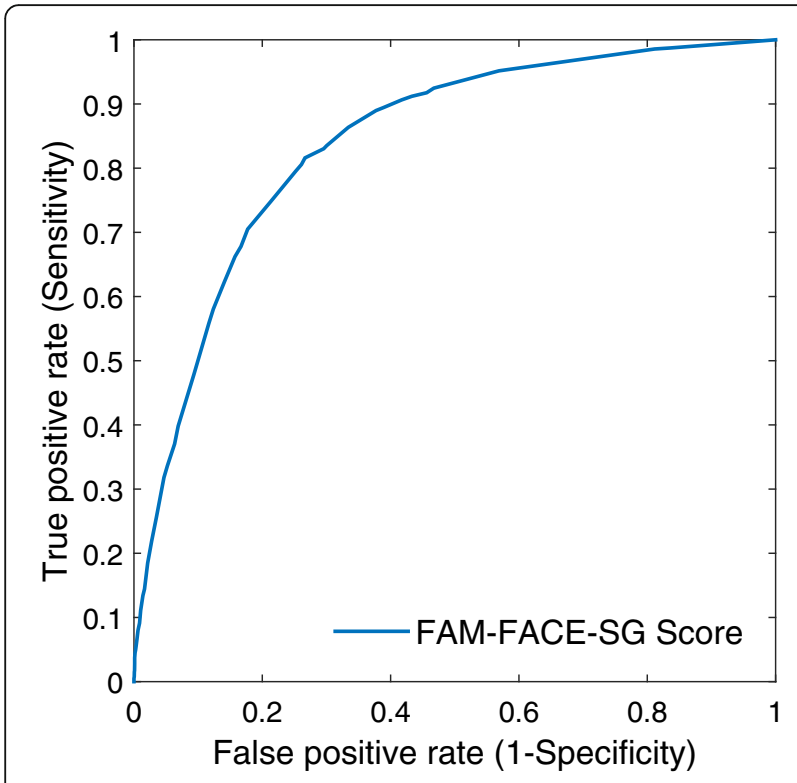

Fig. 2 The receiver operating characteristic curve for the FAM-FACE-SG score

collection. It also makes the use of the tool sustainable as it requires minimal technical support.

The key to driving implementation and adoption is automation. In deriving the FAM-FACE-SG score, we ensured that all variables can be retrieved from our EHR system near real-time to be automated into a risk score that is clinically useful to clinicians and case managers. Relevant facts and dimensions are compiled using stored procedures in our enterprise data mart before the risk score is tabulated using compiled tables. The data mart system is then scheduled to run every midnight \& produce risk scores for patients admitted that same day. The risk scores are available "near real-time" to clinicians and case managers on the day after the admission day.

\section{Limitations}

We believe that the FAM-FACE-SG score is a practical risk score that has implementation potential in other health systems. However, there are limitations to our study.

Table 4 Discriminatory values for FAM-FACE-SG score and the LACE index

\begin{tabular}{lll}
\hline & FAM-FACE-SG & LACE \\
\hline AUC (95\% Cl) & $0.839(0.825-0.853)$ & $0.761(0.745-0.777)$ \\
Cutoff score & 13 & 8 \\
Sensitivity (95\% Cl) & $81.6 \%(79.5 \%-83.7 \%)$ & $65.8 \%(63.2 \%-68.4 \%)$ \\
Specificity (95\% Cl) & $73.3 \%(72.2 \%-74.4 \%)$ & $76.2 \%(75.2 \%-77.3 \%)$ \\
PPV (95\% Cl) & $37.9 \%(36.1 \%-39.8 \%)$ & $36.3 \%(34.3 \%-38.2 \%)$ \\
NPV (95\% Cl) & $95.2 \%(94.6 \%-95.8 \%)$ & $91.5 \%(90.8 \%-92.3 \%)$ \\
\hline
\end{tabular}

$A U C$ area under the curve, $\mathrm{Cl}$ confidence interval, $P P V$ positive predictive value, NPV negative predictive value
First, variables in our dataset are restricted to those routinely collected in our administrative and clinical database. We did not include predictors such as caregiver availability and function which would require additional effort in data collection and therefore impractical for incorporation into a risk score. Second, we did not exclude patients who might have deceased after index hospital discharge. This may have introduced biases in model building. In fact, end of life patients who are identified as high risk for frequent hospital admission can be appropriately referred to hospice programs to reduce their readmission risk and morbidity from unnecessary hospital procedures. Third, unavailability of the required variables in calculating the FAM-FACE-SG score may prevent the use of the score in other healthcare systems. However, the method of score derivation could be adapted to customize the score in different systems.

\section{Conclusions}

We derived a frequent hospital admission risk score (FAMFACE-SG) incorporating demographics, indicators of socioeconomic status, prior healthcare utilization, markers of acute illness burden and markers of chronic illness burden. The FAM-FACE-SG score had excellent discriminative ability with an AUC of 0.839 for prediction of frequent hospital admission risk. It has strong potential for implementation to provide near real-time prediction. A feasibility study is required to study its effectiveness to reduce hospital admissions and external validation is necessary to ensure reproducibility in other health systems.

\section{Abbreviations}

AUC: Area under the curve; CCl: Charlson comorbidity index; Cl: Confidence interval; CIRB: Centralized institutional review board; ED: Emergency department; EHR: Electronic health record; ICD: International classification of diseases; NPV: Negative predictive value; OR: Odds ratio; PN: Patient navigator; PPV: Positive predictive value; RHS: Regional health systems; ROC: Receiver operating characteristic; SD: Standard deviation; SGH: Singapore General Hospital

\section{Acknowledgements}

Not applicable.

\section{Funding}

This study was supported by Singapore Ministry of Health $(\mathrm{MOH})$ Health Services Research New Investigator Grant HSRNIG14nov002 and the SingHealth Foundation Health Services Research (Ageing) Grant SHF/ HSRAg004/2015. The funders had no role in study design, data collection and analysis, decision to publish, or preparation of the manuscript.

\section{Availability of data and materials}

The data used in this paper are owned by the Singapore General Hospital. Data are available from the Singapore General Hospital for researchers who meet the criteria for access to confidential data.

\section{Authors' contributions}

LLL and NL conceived and designed the experiments. LLL, NL and SW analyzed the data. LLL, NL, KHL, MEHO, SW, XJ and JT wrote the paper. LLL and NL supervised the study. All authors read and approved the final manuscript. 


\section{Consent for publication}

Not applicable.

\section{Ethics approval and consent to participate}

This study was approved by SingHealth Centralized Institutional Review Board (CIRB 2015/2696) with waiver of informed consent.

\section{Publisher's Note}

Springer Nature remains neutral with regard to jurisdictional claims in published maps and institutional affiliations.

\section{Author details}

'Department of Family Medicine \& Continuing Care, Singapore General Hospital, Singapore, Singapore. ${ }^{2}$ Family Medicine Program, Duke-NUS Medical School, Singapore, Singapore. ${ }^{3}$ Health Services Research Centre, Singapore Health Services, Singapore, Singapore. ${ }^{4}$ Centre for Quantitative Medicine, Duke-NUS Medical School, Singapore, Singapore. ${ }^{5}$ Department of Emergency Medicine, Singapore General Hospital, Singapore, Singapore. ${ }^{6}$ Health Services and Systems Research, Duke-NUS Medical School, Singapore, Singapore. IIntegrated Health Information Systems, Singapore, Singapore. ${ }^{8}$ Department of Rheumatology and Immunology, Singapore General Hospital, Singapore, Singapore.

Received: 1 December 2016 Accepted: 5 April 2017

Published online: 08 April 2017

\section{References}

1. Kansagara D, et al. Risk prediction models for hospital readmission: a systematic review. JAMA. 2011;306(15):1688-98.

2. Low LL, Liu N, Wang S, Thumboo J, Ong ME, Lee KH. Predicting frequent hospital admission risk in Singapore: a retrospective cohort study to investigate the impact of comorbidities, acute illness burden and social determinants of health. BMJ Open. 2016;6(10):e012705

3. "Readmissions Reduction Program." [Online]. Available: https://www.cms. gov/medicare/medicare-fee-for-service-payment/acuteinpatientpps/ readmissions-reduction-program.html. Accessed 5 May 2016.

4. Chechulin Y, Nazerian A, Rais S, Malikov K. Predicting patients with high risk of becoming high-cost healthcare users in Ontario (Canada). Healthc Policy. 2014;9(3):68-79.

5. Rais S, Nazerian A, Ardal S, Chechulin Y, Bains N, Malikov K. High-cost users of Ontario's healthcare services. Healthc Policy. 2013;9(1):44-51.

6. Robst J. Developing models to predict persistent high-cost cases in Florida medicaid. Popul Health Manag. 2015;18(6):467-76.

7. Saxena N, You AX, Zhu Z, Sun Y, George PP, Teow KL, et al. Singapore's regional health systems-a data-drivenperspective on frequent admitters and cross utilization of healthcare services in three systems. Int J Health Plann Manage. 2017;32(1):36-49.

8. Low LL, Tay WY, Ng MJM, Tan SY, Liu N, Lee KH. Frequent hospital admissions in Singapore: clinical risk factors and impact of socioeconomic status. Singapore Med J. 2016;2016:1-16.

9. Raven MC, Billings JC, Goldfrank LR, Manheimer ED, Gourevitch MN. Medicaid patients at high risk for frequent hospital admission: real-time identification and remediable risks. J Urban Heal. 2009;86(2):230-41.

10. Black JT. Learning about 30-day readmissions from patients with repeated hospitalizations. Am J Manag Care. 2014;20(6):e200-7.

11. Mills RM. The heart failure frequent flyer: an urban legend. Clin Cardiol. 2009;32(2):67-8

12. "Singapore Beats Hong Kong in Health Efficiency: Southeast Asia." [Online]. Available: http://www.bloomberg.com/news/articles/2014-09-18/singaporebeats-hong-kong-in-health-efficiency-southeast-asia. Accessed 5 May 2016.

13. Lai L. "SingHealth's 'navigators' steer patients through health-care system". The Straits Times; 2014. http://www.straitstimes.com/singapore/health/ singhealths-navigators-steer-patients-through-health-care-system.

14. Low LL, Vasanwala FF, Ng LB, Chen C, Lee KH, Tan SY. Effectiveness of a transitional home care program in reducing acute hospital utilization: a quasi-experimental study. BMC Heal Serv Res. 2015;15:100.

15. Lee $\mathrm{KH}$, et al. Transitional care for the highest risk patients: findings of a randomised control study. Int J Integr Care. 2015;15(22):e039.

16. Low LL, et al. Predicting 30-Day readmissions: performance of the LACE index compared with a regression model among general medicine patients in Singapore. Biomed Res Int. 2015;2015:169870.
17. Zhou H, Della PR, Roberts P, Goh L, Dhaliwal SS. Utility of models to predict 28-day or 30-day unplanned hospital readmissions: an updated systematic review. BMJ Open. 2016;6(6):e011060.

18. van Walraven $C$, et al. Derivation and validation of an index to predict early death or unplanned readmission after discharge from hospital to the community. Can Med Assoc J. 2010;182(6):551-7.

19. Shadmi E, Flaks-Manov N, Hoshen M, Goldman O, Bitterman H, Balicer RD. Predicting 30-day readmissions with preadmission electronic health record data. Med Care. 2015;53(3):283-9.

20. Tan SY, Low LL, Yang Y, Lee KH. Applicability of a previously validated readmission predictive index in medical patients in Singapore: a retrospective study. BMC Heal Serv Res. 2013;13(1):366.

21. Charlson ME, Pompei $P$, Ales KL, Mackenzie CR. A new method of classifying prognostic comorbidity in longitudinal studies: development and validation. J Chronic Dis. 1987:40(5):373-83.

22. Deyo RA, Cherkin DC, Ciol MA. Adapting a clinical comorbidity index for use with ICD-9-CM administrative databases. J Clin Epidemiol. 1992;45(6):613-9.

23. Quan $\mathrm{H}$, et al. Coding algorithms for defining comorbidities in ICD-9-CM and ICD-10 administrative data. Med Care. 2005;43(11):1130-9.

24. Howell S, Coory M, Martin J, Duckett S. Using routine inpatient data to identify patients at risk of hospital readmission. BMC Health Serv Res. 2009;9:96.

25. "Ministry of Health Singapore: Medifund." [Online]. Available: https://www. moh.gov.sg/content/moh_web/home/costs_and_financing/schemes_ subsidies/Medifund.html. Accessed 5 May 2016.

26. Low LL, Liu N, Wang S, Thumboo J, Ong MEH, Lee KH. Predicting 30-day readmissions in an asian population: building a predictive model by incorporating markers of hospitalization severity. PLoS One. 2016;11(12):1-16.

27. Cardinal RN, Savulich G, Mann LM, Fernández-Egea E. Association between antipsychotic/antidepressant drug treatments and hospital admissions in schizophrenia assessed using a mental health case register. NPJ Schizophr. 2015;1:15035.

28. Warnke I, Nordt C, Moock J, Kawohl W, Rössler W. Antidepressants: relationship to the time to psychiatric readmission and probability of being in hospital in depressive patients. Front Public Heal. 2014;2:40.

29. Heldeweg MLA, et al. A novel cardiovascular risk stratification model incorporating ECG and heart rate variability for patients presenting to the emergency department with chest pain. Crit Care. 2016;20(1):1-9.

30. Low LL, Wah W, Ng MJ, Tan SY, Liu N, Lee KH. Housing as a Social Determinant of Health in Singapore and its association with readmission risk and increased utilization of hospital services. Front Public Health. 2016:4:109.

\section{Submit your next manuscript to BioMed Central and we will help you at every step:}

- We accept pre-submission inquiries

- Our selector tool helps you to find the most relevant journal

- We provide round the clock customer support

- Convenient online submission

- Thorough peer review

- Inclusion in PubMed and all major indexing services

- Maximum visibility for your research

Submit your manuscript at www.biomedcentral.com/submit
C Biomed Central 